Article

\title{
Bloch Oscillations Due to Quantum Domain Breathing in One-Dimensional Electronic Photoinduced Phase Transitions
}

\author{
Kaoru Iwano \\ High Energy Accelerator Research Organization (KEK), Institute of Materials Structure Science, \\ Graduate University for Advanced Studies, 1-1 Oho, Tsukuba 305-0801, Japan; kaoru.iwano@kek.jp
}

Received: 9 May 2019; Accepted: 13 June 2019; Published: 16 June 2019

\begin{abstract}
We theoretically predict a novel oscillation that will be observed during the dynamical processes of one-dimensional electronic photoinduced phase transitions. This oscillation is considered to be a breathing mode of a quantum domain of a photoinduced phase in the background of the initial phase. When the initial phase is sufficiently stable, being far apart from the phase boundary, the domain feels a constant attractive force depending on its size or the distance between the two domain walls. This fact allows an interpretation that this oscillation is essentially the same as a so-called Bloch oscillation seen for the Stark ladder.
\end{abstract}

Keywords: photoinduced electronic phase transitions; Bloch oscillation; Stark ladder

\section{Introduction}

The phenomena of photoinduced electronic phase transitions (PIPT) have been attracting much attention for decades [1-6]. In particular, their ultrafast aspects are important [7-10], because the dynamics are considered to be mainly related to the electronic degrees of freedom. Their time scales are of the order of several femto to several ten femto seconds, which are roughly the inverse of electron transfer energies. The actual materials are, for instance, tetrathiafulvalene-p-chloranil (TTF-CA) as a neutral-ionic transition system [7,11], one of the MX chains, $\left[\mathrm{Pd}(\mathrm{chxn})_{2} \mathrm{Br}_{\mathrm{Br}} \mathrm{Br}_{2}\right.$ with $\mathrm{M}=\mathrm{Pd}$ and $\mathrm{X}=\mathrm{Br}[9,10]$, which are both quasi-one-dimensional systems, and $\alpha$-(BEDT-TTF) ${ }_{2} \mathrm{I}_{3}$ (BEDT-TTF: bis(ethylenedithio)-tetrathiafulvalene) as a quasi-two-dimensional molecular solid [8]. To describe such systems, we assume a model, which consists of only electronic degrees of freedom. The actual examples are Hubbard models, extended Hubbard models, and other models that are appropriately modified depending on each system [12-18]. What is crucial here is that the Hilbert space associated with the model should have some special states originating from another phase embedded in a low-energy region that can be accessed by photoexcitation. We call it Hilbert space anomaly. In our scenario, we think that such states are excited by light and the system is inevitably changed into another phase.

Here, we must be cautious about the following point. In Figure 1a, we draw a situation very schematically. The state $|g\rangle$ is the ground state from which we start a photodynamics, while $\left|g^{\prime}\right\rangle$ is that for another phase. In a finite-size cluster, their energies can be close to each other as drawn here. However, in the thermodynamic limit, the energy difference becomes infinite, which means that the photoexcitation with modest photon energy from $|g\rangle$ to $\left|g^{\prime}\right\rangle$ never occurs. Furthermore, the matrix element of photoexcitation, $\left\langle g^{\prime}|\hat{J}| g\right\rangle$, where $\hat{J}$ is the electric current operator, is considered to be very small, assuming that they have completely different electronic configurations. Note that $\hat{J}$ is assumed to have a short-range nature, like the model itself. For these reasons, the direct transition from $|g\rangle$ to $\left|g^{\prime}\right\rangle$ is strictly prohibited in typical situations, and we think of another situation as illustrated in Figure 1b. Again, we assume a model such as a Hubbard model or an extended Hubbard model 
with short-range interactions. In such models, the photoexcitation is described as a charge transfer over only a few sites. From here on, we restrict ourselves to the simplest case where only one site transfer is allowed, because the generalization is straightforward. Such a situation is realized with a model in which only the nearest-neighbor electron hopping is assumed for the Hamiltonian and the electric current operator. The excited electron and the hole are located at neighboring sites just after photoexcitation. We consider this pair as a transition core of PIPT and think that this core evolves into a spatially extended region, that is, a domain, as shown in Figure 1b. We emphasize that this domain can be located anywhere, which property is nothing but a translational invariance. The true state is a linear combination of individual states at different positions and keeps quantum coherence.

(a)

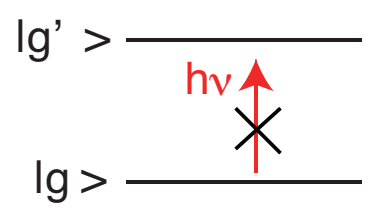

(b)

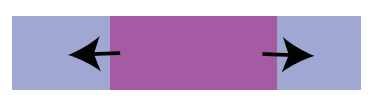

(c)

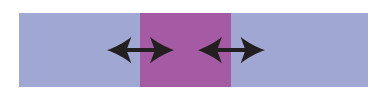

Figure 1. Scenarios of photoinduced electronic phase transitions (PIPTs). (a) Direct transition from the ground state of the initial phase to that of another phase. In a bulk system, this is strictly prohibited. (b) Domain formation on the phase boundary and (c) the formation of a small domain in situations far from the phase boundary. In (b), the domain grows persistently in the absence of dissipation, while the growth saturates and a breathing oscillation remains in (c).

So far, we discussed the growth of such quantum domain $[13,14]$. Particular attention was paid to the cases in which the initial state is in the vicinity of the phase boundary. In those cases, the domain grows most effectively once it is created with sufficient energy. Such growth is associated with one of its internal degrees of freedom, that is, the spatial size. In one dimension, this size is identical to the distance between the two domain walls on both sides. In the higher dimensional cases, on the other hand, the most fundamental degree of freedom is the radius of a domain, although the degrees of the shape make the description more complicated. As is already mentioned, the quantum domain can be dispersive. Such a feature is associated with its center of gravity, as was discussed in the past studies [14,15]. Furthermore, the domain growth does not continue forever [16]. Briefly speaking, the quantum domain is affected by other degrees of freedom, for instance, the phonons, which provide incoherence to the growth process, and we expect that they possibly hinder the growth, as a similar mechanism argued for quantum friction [19].

In this article, we focus on a more general case. We start the dynamics after the photoexcitation from an initial state, which is chosen as a certain point in the ground-state phase diagram. We assume that this point is not close to the phase boundary. In these cases, true PIPTs are not expected to occur. However, we think that it is possible to observe a precursor. Here, the precursor is defined as a quantum domain with a small spatial extension. One of its detections is of course via the difference spectrum between the spectra before and after the photoexcitation. The problem will be a small signal because of the relatively small converted fraction. We here propose another detection, which is via the oscillation typical to the small quantum domain. As shown in Figure 1c, this oscillation is interpreted as a breathing mode of the domain. It has at least two advantages in the detection. The first advantage is that the frequency can be located within the optical gap, which makes the identification relatively feasible. As the second advantage, when the system is one-dimensional, the oscillation is equivalent to the so-called Bloch oscillation found for a wave packet in a uniform field, namely, in a Stark ladder [20-23], which means that the oscillation frequency is basically identical to the step energy, that is, the energy difference corresponding to the one-site growth. Such features are argued in detail in the following sections. 


\section{Model and Method}

We employ the same model that we used in past studies. This model has been used for describing TTF-CA [12,24-27], and its model hamiltonian in the absence of lattice deformation is

$$
H=-t_{0} \sum_{l \sigma}\left(e^{-i(e a / \hbar c) A(t)} C_{l+1 \sigma}^{+} C_{l \sigma}+\text { H.c. }\right)+U \sum_{l} n_{l \uparrow} n_{l \downarrow}+V \sum_{l} n_{l} n_{l+1}+\Delta_{e f f} \sum_{l=e v e n} n_{l},
$$

where $C_{l \sigma}$ is the electron operator at the $l$ th site with spin $\sigma$, and $n_{l \sigma}$ is its number operator with $n_{l}=n_{l \uparrow}+n_{l \downarrow}$. We adopt the periodic boundary condition. Here, we place TTF (CA) molecules at the odd- (even-) numbered sites. The effect of light is incorporated via the Peierls phase, namely, the extra phase factor in the first term, with $A(t),-e$, and $a$ being the vector potential of light, the electronic charge $(e=|e|)$, and the lattice constant, respectively. TTF -CA is well known to have two fundamental phases, which are the neutral phase and the ionic phase, as illustrated in Figure 2 [28-35]. In the neutral phase, each molecule remains electrically neutral, while a charge transfer occurs from the donor (D) to the acceptor (A), leading to a chain of $\mathrm{D}^{+}$and $\mathrm{A}^{-}$. From here on, we assume that the initial state is in the neutral phase. In this case, the excitation energies in the localized limit are $V+n_{e x}\left(2 V+\Delta_{e f f}-U\right)$ with $n_{e x}$ being the number of electron excitations, which are nearly degenerate around the phase boundary, i.e., $2 \mathrm{~V}+\Delta_{\text {eff }}=U$. The parameters appropriate for TTF-CA are estimated as $U=2.4080 \mathrm{eV}$, $V=1.070 \mathrm{eV}$, and $t_{0}=0.17 \mathrm{eV}[13,36,37]$. The remaining parameter, $\Delta_{e f f}$, takes a value close to $0.27 \mathrm{eV}$, which is the value on the phase boundary. In this article, we mainly assume much larger values because we are interested in cases that are rather apart from the phase boundary. In the actual case, lattice deformations such as the molecular dimerization are known to occur in the course of the neutral-to-ionic transition, although we do not argue them here because we are interested in the early stage of dynamics before the lattice deformations occur.

\section{(a) Neutral Phase(N)}

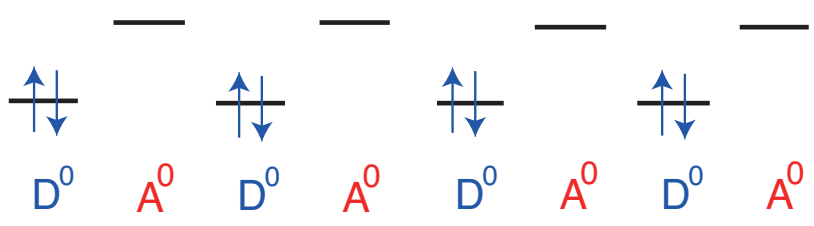

(b) Ionic Phase(I)

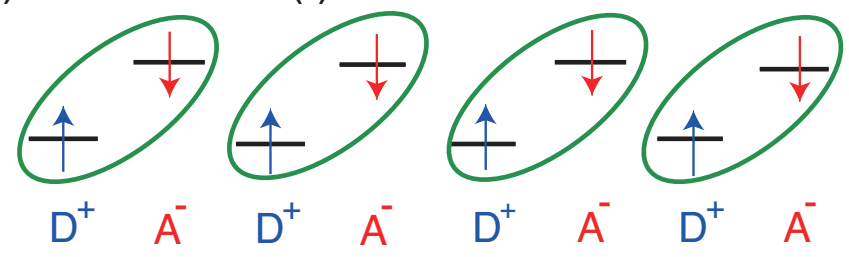

Figure 2. Schematics for a neutral-ionic-transition system. (a) The neutral phase and (b) the ionic phase. $\mathrm{D}$ and A mean a donor and an acceptor, which correspond to TTF and CA, respectively, for TTF-CA. The green oval indicates molecular dimerization.

To discuss dynamical behaviors, namely, time evolutions after photoexcitation, we first determine the ground state $|g\rangle$ by exact diagonalization and next perform exact time-dependent calculation, using a $N$-site ring. For the extended Hubbard model, we choose $N=16$. The vector potential along the chain direction, $A(t)$, is expressed by the electric field along the same direction, $E(t)$, as

$$
A(t)=-c \int_{0}^{t} d t^{\prime} E\left(t^{\prime}\right)
$$


In this work, we assume a step function for the envelope of $E(t)$, as

$$
E(t)=E_{0} \theta(t) \theta\left(\tau_{d}-t\right) \sin \left(\omega_{c} t\right),
$$

where $E_{0}, \omega_{c}$, and $\tau_{d}$ are the amplitude of the uniform electric field, the central frequency, and the duration time of the pulse, respectively, and $\theta(t)$ is the step function. Using Equations (2) and (3), the explicit functional form of $A(t)$ for $t<\tau_{d}$ is

$$
A(t)=c E_{0}\left(\cos \left(\omega_{c} t\right)-1\right) / \omega_{c},
$$

and $A(t)=A\left(\tau_{d}\right)$ for $t \geq \tau_{d}$. Compared with other pulse forms, the present form has an advantage that the check of the calculation is easy because the total energy is conserved after the pulse, i.e., $t \geq \tau_{d}$. The method is based on a Taylor expansion of the time evolution operator [38]. We include the terms up to 10th, which preserve the norm of the state vector with practically sufficient precision during the time region treated in this article. Throughout this article, the unit of time is $\hbar / \mathrm{eV} \sim 0.66 \mathrm{fs}$. In addition to this model, we also use an effective model, that is, a domain model. The treatment of this model is straightforward and allows us to calculate time evolutions in huge systems.

For the original model in Equation (1), we also calculate an ordinary optical conductivity spectrum, which is expressed as

$$
\sigma(\omega)=\frac{\gamma}{\omega N} \sum_{\mu}|\langle\mu|\hat{J}| g\rangle|^{2} \frac{1}{\left(\omega-E_{\mu}+E_{g}\right)^{2}+\gamma^{2}}
$$

where $E_{g}$ is the ground state energy, and $|\mu\rangle$ is the eigenstate associated with the energy eigenvalue $E_{\mu}$. Here, the artificial broadening $\gamma$ is set to $0.1 t_{0}$. The current operator, $\hat{J}$, takes the ordinary definition as

$$
\hat{J}=-i e a t_{0} \sum_{l \sigma}\left(C_{l+1 \sigma}^{\dagger} C_{l \sigma}-\text { H.c. }\right) .
$$

We calculate the optical conductivity by the method of a continued fraction [39].

\section{Results}

\subsection{Extended Hubbard model}

In Figure 3, We show the results of time-dependent calculations for $\Delta_{\text {eff }}=0.28 \mathrm{eV}$. These results correspond to the case slightly apart from the phase boundary, and we expect that the domain grows most effectively in the absence of dissipation. In other words, this is a typical PIPT, in which a macroscopic part of the sample is converted into another phase [40-42]. In Figure 3a, the electron site density at the odd-numbered site (odd site, hereafter) is depicted. Here, $\tau_{d}$ and $\hbar \omega_{c}$ are $30(\hbar / \mathrm{eV})$ and $1.0 \mathrm{eV}$, respectively. The strength of the amplitude of the electric field is set at $e a E_{0}=0.2 \mathrm{eV}$ throughout this subsection. Since the initial state is in the neutral phase, the odd site is initially the charge-rich site. The decrease in the density at the odd site consequently indicates the transition from the neutral state to the ionic state. In experiments, the time dependency is typically observed for the photoinduced reflectivity $(\Delta R / R)$ at a fixed frequency, and the result is transformed into a spectrum via wavelet analyses. Here, we avoid direct calculation of reflectivity and focus on the site density because the latter affects the former very sensitively. By the way, the present system size is limited to 16 sites, which is the reason that the curve of the conversion seems to be rather saturated despite the initial state just on the phase boundary.

In addition to the overall decrease in the site density, we observe an oscillatory component. To analyze the oscillatory component, we fit the smooth background to a function expressed by the green curve and subtract it from the original curve. The extracted component is applied to wavelet analysis, giving a spectrogram appearing in Figure 3b. Finally, we integrate this over the observation time and obtain the frequency distribution (red curve) in Figure 3c. For comparison, we also show 
the ordinary optical conductivity spectrum (blue curve) and find that they are similar to each other. This is understood in the following way. The general form of the time-dependent state vector is $|\Psi(t)\rangle=\sum_{\mu} a_{\mu} e^{-i \omega_{\mu} t}|\mu\rangle$, where $\mid \mu>$ and $\omega_{\mu}$ are the eigenstate and its eigenenergy, respectively. Note that the coefficient $a_{\mu}$ are constant after the pulse end, namely, $t \geq \tau_{d}$. When we take the matrix element as $\left\langle\Psi(t)\left|n_{l}\right| \Psi(t)\right\rangle$, the terms like $a_{\mu^{\prime}}^{*} a_{\mu} e^{-i\left(\omega_{\mu}-\omega_{\mu^{\prime}}\right) t}$ appear, and the combinations of $|\mu\rangle$ and $\left|\mu^{\prime}\right\rangle$ as $|g\rangle$ and any excited state give the oscillations corresponding to the optical conductivity spectrum. Here, we make two remarks on the peaks around zero energy in the optical conductivity. Firstly, the enhancement at zero energy originates from the prefactor of $1 / \omega$ in the optical conductivity formula in Equation (5). Secondly, the peaks at less than $0.25 \mathrm{eV}$ are attributable to the spin excitations from the state of the ionic phase. As was mentioned in Ref. [13], the state of the ionic phase exists energetically slightly higher than that of the neutral phase, that is, the ground state of this case. According to a past study [43], the spin excitations from the ionic state are optically active due to the site-alternate potential appearing as the last term of Equation (1). Anyway, the appearance of such low energy peaks is due to a size effect, and we neglect them in the following.

(a)

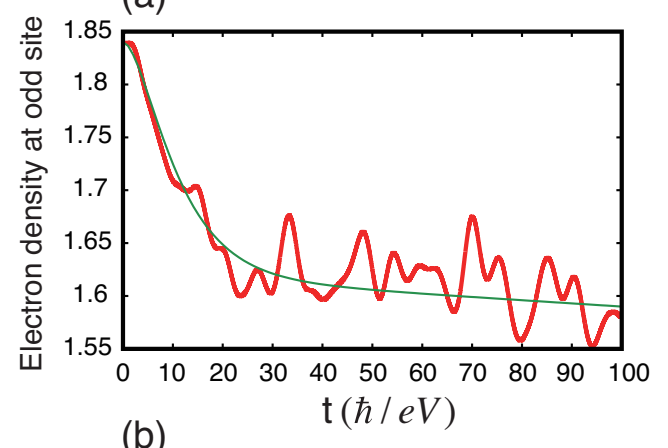

(b)

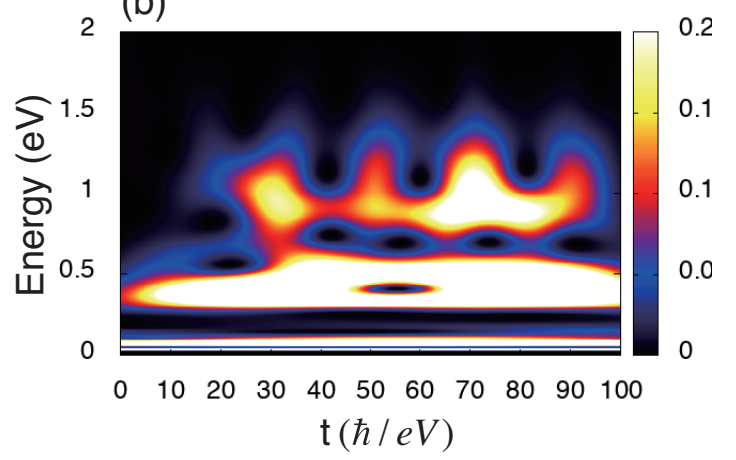

0.2

1 (c)

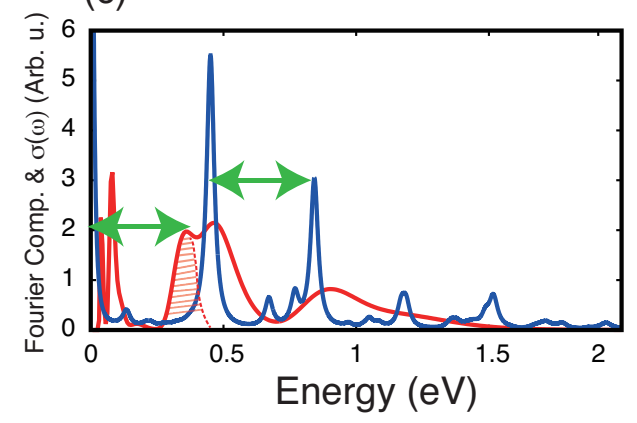

Figure 3. Dynamical behaviors for $\Delta_{e f f}=0.28 \mathrm{eV}$ during and after the pulse excitation with $\hbar \omega_{c}=1.0 \mathrm{eV}$ and $\tau_{d}=30(\hbar / \mathrm{eV})$. (a) The site density at the odd-numbered site; (b) a spectrogram obtained from $(\mathbf{a})$; and (c) the time integration of (b) (red line) and optical conductivity (blue line). The two green arrows in (c) show that the energy difference is the same at the two parts.

Meanwhile, we carefully inspect Figure $3 \mathrm{c}$ and notice an oscillatory component that is not seen in the optical conductivity, which is located around $0.40 \mathrm{eV}$ (hatched region), slightly below the lowest peak of the optical conductivity. We consider this contribution as originating from the term as $|\mu\rangle$ and $\left|\mu^{\prime}\right\rangle$ being the first and second optically excited states that correspond to the first and second peaks in the optical conductivity, respectively. The energy difference between the peaks is around $0.4 \mathrm{eV}$, which is almost the same as the energy of the new oscillatory component, as specified by the horizontal arrows. The appearance of this component is quite natural because the coherence between the excited states is maintained in our calculation that assumes no dissipation. Regarding this new oscillation, we add one more remark. We easily notice a repeated pattern in the region of $t \geq 30$ $(\hbar / \mathrm{eV})$ and $E \geq 0.5 \mathrm{eV}$ of Figure $3 \mathrm{~b}$. Checking the period of this time structure, we judge that this is another manifestation of the new oscillation. As the last comment to Figure 3, the peak distribution 
of the optical conductivity is rather sparse. Since it is a continuum in an infinite system on the phase boundary, this sparse distribution should be interpreted as due to the finite size effect.

We next argue the cases of which the initial states are far from the phase boundary. In Figure 4, we summarize the results calculated for $\Delta_{e f f}=0.50 \mathrm{eV}$ and $\hbar \omega_{c}=1.5 \mathrm{eV}$. The larger value for $\Delta_{e f f}$ lowers the site energy of the odd sites, leading to the stabilization of the neutral phase. Again, we show three figures that are analogous to those in Figure 3. In addition, in this case, we recognize an oscillational component that is absent in the optical conductivity, around $0.5 \mathrm{eV}$. We again attribute this to the energy difference between the first peak and the second energy band in the optical conductivity, as specified by the horizontal arrows. We emphasize that the peak positions in the spectrum are almost free from the size effect because the larger order parameter in this case makes the state rather localized [13]. We, therefore, assure that the energy of the new oscillatory component is well below the optical gap of about $1 \mathrm{eV}$, which suggests the feasibility of its detection. At this point, readers might have a question related to the covered energy region. Namely, the present duration time is $30(\hbar / \mathrm{eV})$, of which the inverse is roughly $0.03 \mathrm{eV}$. Even if we multiply it by $2 \pi$, it becomes only $0.2 \mathrm{eV}$, which is much smaller than the concerned energy difference. We note that the present pulse form is a step function and that the covered energy region extends widely obeying a function like $1 /\left(E-\hbar \omega_{c}\right)^{2}$. We confirm this region in Figure $4 \mathrm{~d}$, which shows energy distribution in the excited states immediately after the pulse, with the case of $\Delta_{e f f}=0.50 \mathrm{eV}$ as an example. We find that not only the directly excited region around $\hbar \omega=1.5 \mathrm{eV}$ but also the state of the first peak is substantially excited due to this wide extension. We also see at least two oscillatory components at $0.25 \mathrm{eV}$ and $0.2 \mathrm{eV}$. While detailed analyses are still required, we tentatively attribute the former to the interband coherence between the second energy band and the third energy band and the latter to the intraband coherence within the second energy band.

(a)

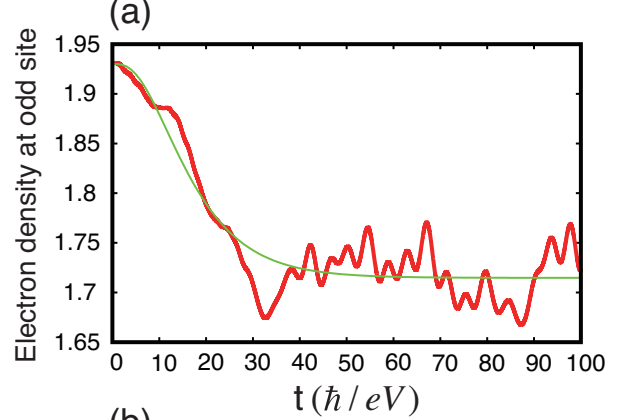

(b)

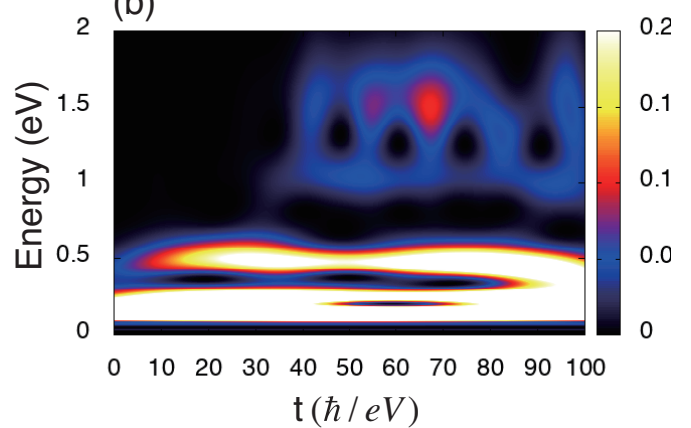

(c)

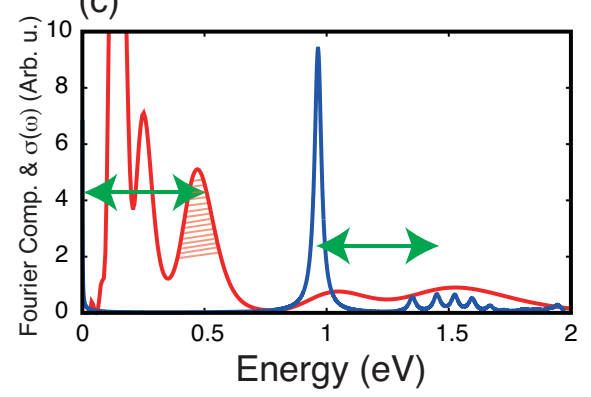

(d)

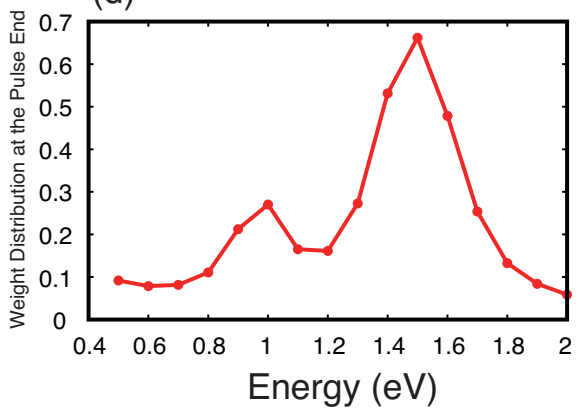

Figure 4. (a-c) Same as Figure 3, but for $\Delta_{e f f}=0.50 \mathrm{eV}$ and $\hbar \omega_{c}=1.5 \mathrm{eV}$; (d) weight distribution at the pulse end, i.e., $t=\tau_{d}$.

Almost the same feature is also confirmed in a much more localized case with $\Delta_{\text {eff }}=0.7 \mathrm{eV}$. In Figure 5, we again show the calculated results, which are obtained with $\hbar \omega_{c}=1.2 \mathrm{eV}$. The extra peak appears around $0.7 \mathrm{eV}$, which coincides with the energy difference between the first and second peaks in the optical conductivity. From these three cases, we judge that the coincidence is not accidental 
and that the newly appearing oscillatory components originate from the coherence between the excited states.

(a)

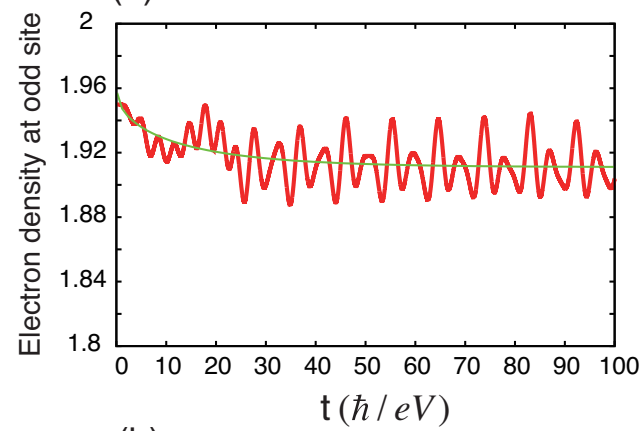

(b) (c)

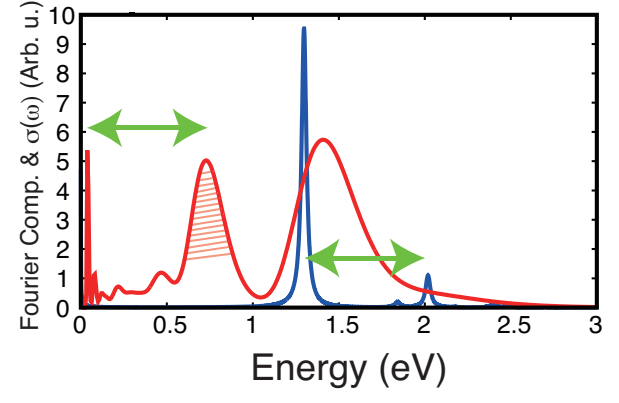

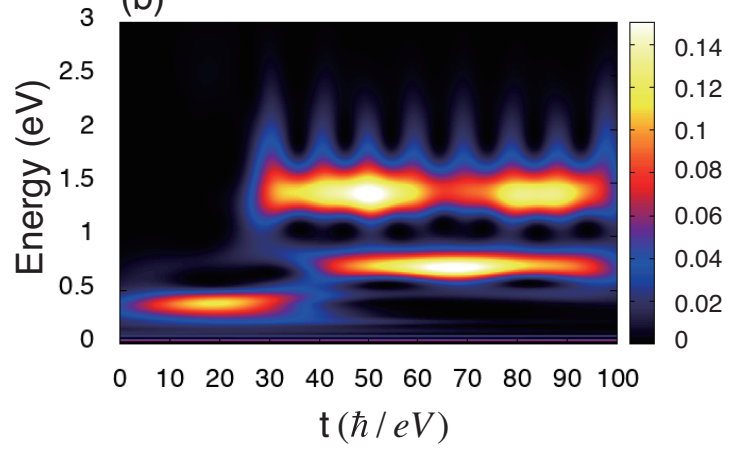

Figure 5. (a-c) Same as Figure 3, but for $\Delta_{e f f}=0.70 \mathrm{eV}$ and $\hbar \omega_{c}=1.2 \mathrm{eV}$.

\subsection{Domain Model}

As is already mentioned, the treatment based on the extended Hubbard model has a disadvantage of a restricted system size. To understand the dynamical behaviors depending on $\Delta_{\text {eff }}$, the use of the domain model [12-14] is considered to be effective. The domain model is defined as

$$
H_{e f f}=-t_{0} \sum_{l_{D}, p}\left\{\left(\left|l_{D}, p+1\right\rangle+\left|l_{D}-1, p+1\right\rangle\right)\left\langle l_{D}, p\right|+\text { h.c. }\right\}+\sum_{l_{D}, p} E(p)\left|l_{D}, p\right\rangle\left\langle l_{D}, p\right|,
$$

where $\left|l_{D}, p\right\rangle$ is the domain state of which the spacial size and the position of the leftmost site are $p$ and $l_{D}$, respectively. The domain energy, $E(p)$, is defined as $E(p)=V+p\left(2 V+\Delta_{e f f}-U\right)$, which consists of the domain internal energy and the domain-wall energy. While this model considers only the domain degree of freedom, it is known to reproduce the properties of the extended Hubbard model close to the phase boundary when the problem is limited to the case in which only one domain exists, that is, the limit of weak excitation. The advantage of this model is, of course, the nature of a one-body problem, which makes calculations in large systems realistic. Another remark related to the treatment of this model is the use of a first-order perturbational theory. Since we assume weak excitation as we already mentioned, this treatment is considered to be appropriate. In actual numerical calculations, we slightly modify the form of $A(t)$ by adding a factor of $\exp (-\gamma t)$ to avoid any accidental divergence in the energy denominator. We set $\gamma$ at $0.002 \mathrm{eV}$, and it is confirmed that the final result is almost the same as the result with $\gamma=0$ when the latter is safely obtained. Furthermore, we restrict our calculation in the subspace of zero total momentum because the momentum of visible or infrared light is negligible. In this case, the effective model is further reduced to that with only $p$, which is expressed as

$$
H_{e f f}=-2 t_{0} \sum_{p}\left((|p+1\rangle\langle p|+\text { h.c. })+\sum_{p} E(p)|p\rangle\langle p| .\right.
$$


We emphasize that the transfer energy is doubled due to the motions of the two domain walls on both sides [14]. Regarding the effect of photoexcitation, it is expressed by the following perturbational term as

$$
H_{e-p h}=E_{0} \theta(t) \theta\left(\tau_{d}-t\right) \sin \left(\omega_{c} t\right)(|p=1\rangle\langle g|+\text { h.c. }) .
$$

Since we use the first-order perturbational theory and only discuss the motions in the excited states, the magnitude of electric field $E_{0}$ is irrelevant in the following.

In Figure 6a, we draw the time evolution of the averaged domain size, which is defined as $\sum_{l_{D}, p} p\left|\left\langle\Psi(t) \mid l_{D}, p\right\rangle\right|^{2}$, with $|\Psi(t)\rangle$ being the time-dependent state vector. $\hbar \omega_{c}$ and $\tau_{d}$ are $1.7 \mathrm{eV}$ and $18(\hbar / \mathrm{eV})$, respectively. As is seen immediately, the oscillatory behavior is observed and its period gradually increases toward the critical point at $\Delta_{e f f}=0.27 \mathrm{eV}$. From a different viewpoint, these are interpreted as the motions of a wave packet along the relative coordinate of the two domain walls, as schematically depicted in Figure 6b.
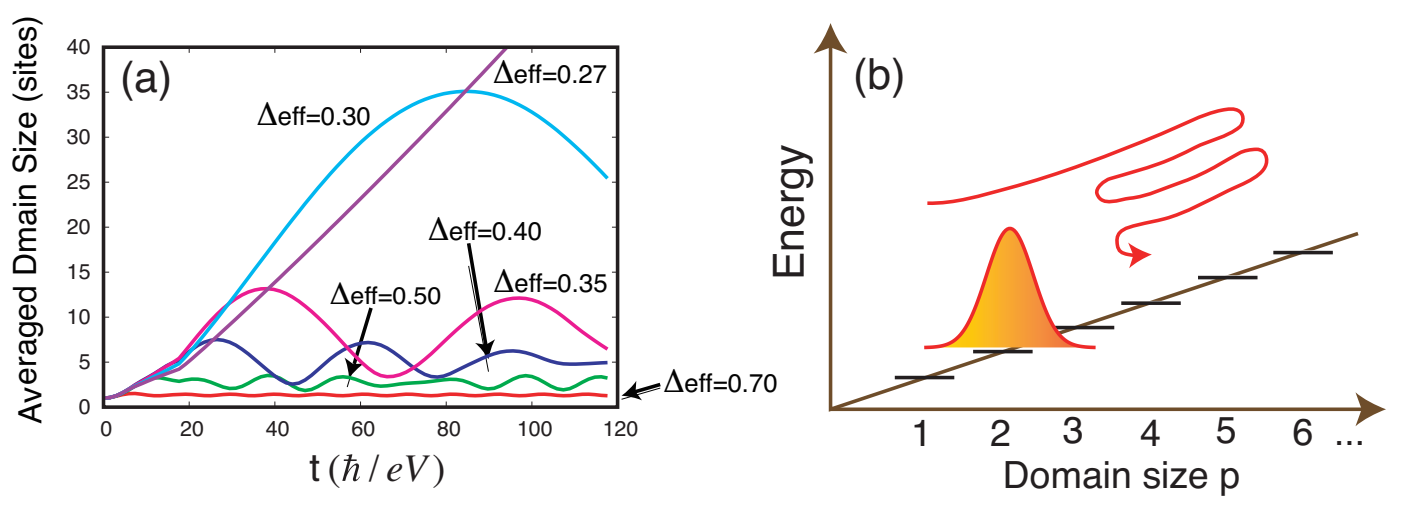

Figure 6. Dynamical behaviors in the domain model. All the results are obtained with $\omega_{\mathcal{c}}=1.7 \mathrm{eV}$ and $\tau_{d}=18(\hbar / \mathrm{eV})$. (a) The time evolutions of the averaged domain size; (b) schematics of a real-space motion of a wave packet defined along the relative coordinate of the two domain walls.

To understand the nature of these oscillations, we perform Fourier analyses, particularly, for $\Delta_{e f f}=0.50 \mathrm{eV}$. In Figure $7 \mathrm{a}$, we show the oscillatory components from which the background components are already subtracted. The red curve is obtained from the corresponding curve in Figure $6 \mathrm{a}$, while the blue curve from the result with $\hbar \omega_{c}$ being as high as $10.2 \mathrm{eV}$. The comparison of these two cases in Figure 7a does not give any meaningful information. However, their Fourier transforms in Figure $7 \mathrm{~b}$ show that the numbers of principal peaks are two and one for the former and the latter, respectively. Closer analyses of the eigenenergies defined as $e(i)$, which appear in Figure $7 \mathrm{c}$, indicate that the two peaks for $\hbar \omega_{c}=1.7 \mathrm{eV}$ are assigned to $e(2)-e(1)$ and $e(3)-e(2)$. As is easily understood from the form of $E(p)$, the present system is considered as a Stark ladder in a uniform field. Only one modification is the boundary condition at the shortest distance between the domain walls, which deforms the differences between the two consecutive eigenenergies at the lower edge from an equidistant structure. In contrast, excitations with higher energies fall within in the ladder more satisfactorily. The single frequency energy confirmed for $\hbar \omega_{c}=10.2 \mathrm{eV}$ corresponds to the unique energy difference between the two consecutive eigenenergies in the high energy limit, although the former energy is deviated from the latter because of the resolution of the Fourier transform. Hereafter we define this energy difference as $E_{d}$.

Such a situation reminds us of a so-called Bloch oscillation that was first proposed for a particle in a crystal under a uniform field [20-23]. In the case of a Bloch oscillation, a periodic motion in real space is expected due to a crystal effect, which is similar to the cases in Figure 6a except that for $\Delta_{e f f}=0.27$. For an infinite Stark ladder, it is well known that $E_{d}$ coincides with the energy difference between the two consecutive sites [22]. Applying this property to the present case, $E_{d}$ is evaluated as $\left(2 V+\Delta_{e f f}-U\right)$ for high-energy excitations. It is also known for the Stark ladder that the period and the amplitude of the oscillation are given as $2 \pi \hbar / E_{d}$ [20] and $4 t_{0} / E_{d}$ [23], respectively. Since the critical 
point is given by $\left(2 V+\Delta_{e f f}-U\right)=0$, both the period and the amplitude diverge toward the critical point. In particular, a more extended period, namely, a smaller oscillation frequency near the phase boundary pushes the signal within the optical gap, because the optical gap remains finite even on the phase boundary [29]. By the way, in the case of an ordinary Bloch oscillation, the wave packet almost keeps its initial form when the form of the initial wave packet is a smooth Gaussian [23]. In the present case, the wave packet gradually loses its original form defined at the pulse end $\left(t=\tau_{d}\right)$, which tendency is attributed to the somewhat complicated form at the pulse end and the boundary condition. As the last remark, we mention the value of $E_{d}$ itself. As is already mentioned, for an infinite Stark ladder, $E_{d}$ is exactly the energy difference between the two consecutive sites. This fact suggests that the frequency of the oscillatory component in high energy excitations gives us the value of $\left(2 V+\Delta_{e f f}-U\right)$ reliably. This contrasts with general cases, where measured quantities are renormalized due to a finite amount of transfer, and it is difficult to estimate the bare values of parameters directly.

(a)
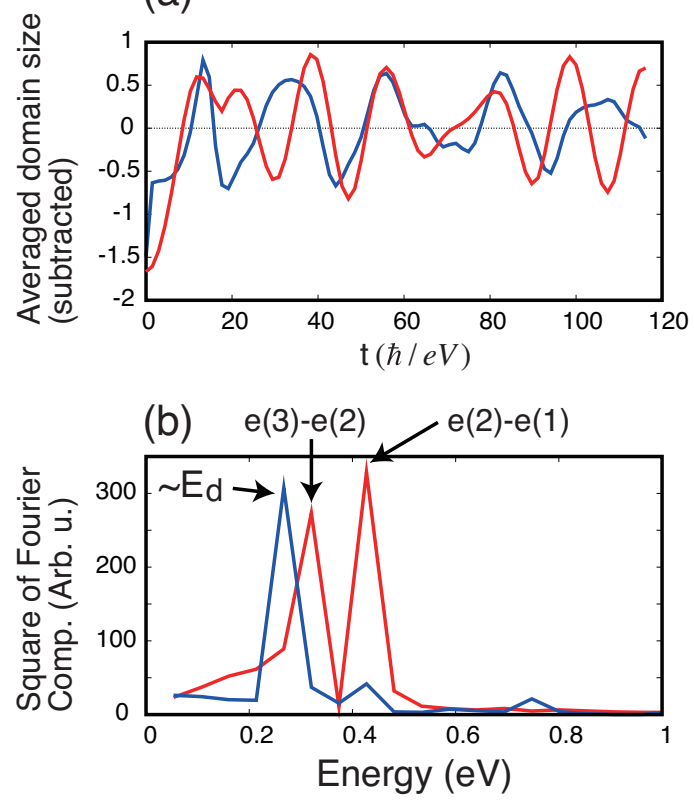

(c)

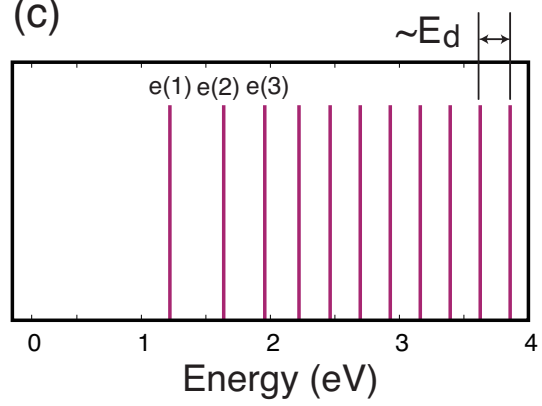

Figure 7. (a) Time evolutions of the averaged domain size from which the background component is subtracted. Both the curves are obtained with $\Delta_{e f f}=0.50 \mathrm{eV}$. The red curve is the same as that in Figure 6a, namely, with $\omega_{c}=1.7 \mathrm{eV}$. The blue curve is calculated assuming $\omega_{c}=10.2 \mathrm{eV}$. (b) Squared magnitudes of Fourier components of each trajectory. Same colors as those in (a) are used for different excitation energies. (c) Eigenenergies measured from the ground state, for $\Delta_{e f f}=0.50 \mathrm{eV}$.

\section{Discussions}

We discussed what we expect to observe in time-resolved measurement in the context of electronic PIPTs. In particular, we focused on the oscillatory components seen in the time-dependent site density in situations where the initial state is located far from the phase boundary. While our calculation is restricted to one dimension, the present results demonstrate that the breathing mode of a domain can be identified as an extra oscillation that is absent in the optical conductivity spectrum. In relation to the experiments, we can choose a target system from various actual materials whose ground states are considered to be far from the phase boundary. For instance, the systematic chart including TTF-CA and other analogous materials [29] will help the exploration of this oscillation. From a theoretical point of view, such oscillation emerges as coherence between optically excited states and is essentially the same as that recognized as Bloch oscillation in a Stark ladder.

As future works, we propose various extensions from the present work. One is the case of higher dimensional systems. In contrast to the one-dimensional case where only the linear size of a domain is relevant, two- and three-dimensional domains can have many shape-related degrees of freedom [18]. 
The exploration of them will be one of the crucial extensions. As another extension, we have concerns about the generation of incoherence. As we already discussed, the concerned oscillation is directly related to the coherence between optically excited states. In other words, the breathing motion of a domain persists until the coherence is lost. Regarding this, the effect of incoherence was discussed in the context of a pure Stark ladder [44]. As the source of incoherence, we think of several candidates such as phonons, magnons, and independent excitations of electrons. The exploration of such interplays is also interesting and is expected to deepen our understanding of PIPTs.

Funding: This work was supported by JST CREST in Japan (Grant No. JPMJCR1661) and the Grant-in-Aid for Scientific Research from JSPS in Japan (Grant No. JP17K05509).

Acknowledgments: The computations were partially performed at Research Center for Computational Science, Okazaki, Japan, and at RCNP and CMC of Osaka University, Osaka, Japan.

Conflicts of Interest: The authors declare no conflict of interest .

\section{References}

1. Toyozawa, Y. Symmetry Breaking Excitonic Instabilities in Deformable Lattice. Physica 1983, 117B\&118B, 23-29. [CrossRef]

2. Toyozawa, Y. Condensation of Relaxed Excitons in Static and Dynamic Phase Transitions. Solid State Commun. 1992, 84, 255-257. [CrossRef]

3. Hanamura, E.; Nagaosa, N. Photo-Induced Structural Changes. J. Phys. Soc. Jpn. 1987, 56, 2080-2088. [CrossRef]

4. Koshihara, S; Adachi, S. Photo-Induced Phase Transition in an Electron-Lattice Correlated System-Future Role of a Time-Resolved X-ray Measurement for Materials Science-. J. Phys. Soc. Jpn. 2006, 75, 011005. [CrossRef]

5. Relaxations of Excited States and Photoinduced Structural Phase Transitions; Nasu, K., Ed.; Springer Series in Solid State Science; Springer: Berlin, Germany, 1997; Volume 124.

6. Yonemitsu, Y.; Nasu, K. Theory of Photoinduced Phase Transitions in Itinerant Electron Systems. Phys. Rep. 2008, 465, 1-60. [CrossRef]

7. Iwai, S.; Ishige, Y.; Tanaka, S.; Okimoto, Y.; Tokura, Y.; Okamoto, H. Coherent Control of Charge and Lattice Dynamics in a Photoinduced Neutral-to-Ionic Transition of a Charge-Transfer Compound. Phys. Rev. Lett. 2006, 96, 057403. [CrossRef] [PubMed]

8. Kawakami, Y.; Fukatsu, T.; Sakurai, Y.; Unno, H.; Itoh, H.; Iwai, S.; Sasaki, T.; Yamamoto, K.; Yakushi, K.; Yonemitsu, K. Early-Stage Dynamics of Light-Matter Interaction Leading to the Insulator-to-Metal Transition in a Charge Ordered Organic Crystal. Phys. Rev. Lett. 2010, 105, 246402. [CrossRef] [PubMed]

9. Matsuzaki, H.; Yamashita, M.; Okamoto, H. Ultrafast Photoconversion from Charge Density Wave State to Mott-Hubbard State in One-Dimensional Extended Peierls-Hubbard System of Br-Bridged Pd Compound. J. Phys. Soc. Jpn. 2006, 75, 123701. [CrossRef]

10. Matsuzaki, H.; Iwata, M.; Miyamoto, T.; Terashige, T.; Iwano, K.; Takaishi, S.; Takamura, M.; Kumagai, S.; Yamashita, M.; Takahashi, R.; et al. Excitation-Photon-Energy Selectivity of Photoconversions in HalogenBridged Pd-Chain Compounds: Mott Insulator to Metal or Charge-Density-Wave State. Phys. Rev. Lett. 2014, 113, 096403. [CrossRef]

11. Okamoto, H.; Ishige, Y.; Tanaka, S.; Kishida, H.; Iwai, S.; Tokura, Y. Photoinduced phase transition in tetrathiafulvalene-p-chloranil observed in femtosecond reflection dpectroscopy. Phys. Rev. B 2004, 70, 165202. [CrossRef]

12. Nagaosa, N.; Takimoto, J. Theory of Neutral-Ionic Transition in Organic Crystals II. Effect of the Intersite Coulomb Interaction. J. Phys. Soc. Jpn. 1986, 55, 2745-2753. [CrossRef]

13. Iwano, K. Direct Photoexcitation of Appreciable Size of Domains without Lattice Motion in Neutral-Ionic Transition Systems. Phys. Rev. Lett. 2006, 97, 226404. [CrossRef] [PubMed]

14. Iwano, K. Detection of Domain Dynamics by High Energy Photons: Proposals in One-Dimensional Photoinduced Phase Transition Systems. Phys. Rev. Lett. 2009, 102, 106405. [CrossRef] [PubMed]

15. Mayr, M.; Horsch, P. Domain-wall excitations and optical conductivity in one-dimensional Wigner Lattices. Phys. Rev. B 2006, 73, 195103. [CrossRef] 
16. Iwano, K. Incoherence induced by phonon excitations in domain-growth dynamics: Role of the domain center-of-gravity motion. Phys. Rev. B 2011, 84, 235139. [CrossRef]

17. Hashimoto, H.; Matsueda, H.; Seo, H.; Ishihara, S. Photo-Induced Dynamics in Charge-Frustrated Systems. J. Phys. Soc. Jpn. 2014, 83, 123703. [CrossRef]

18. Iwano, K. Ultrafast electronic photoinduced phase transition in two-dimensional charge-ordering system. Phys. Rev. B 2015, 91, 115108. [CrossRef]

19. Caldeira, A.O.; Leggett, A.J. Path Integral Approach to Quantum Brownian Motion. Physica 1983, 121A, 587-616. [CrossRef]

20. Bloch, F. Über die Quantenmechanik der Electronen. Zeitschrift Für Phys. 1928, 52, 555-600. [CrossRef]

21. Zener, C. A Theory of the Electrical Breakdown of Solid Dielectrics. Proc. R. Soc. A 1934, 145, 523-529. [CrossRef]

22. Fukuyama, H.; Bari, R.A.; Fogedby, H.C. Tightly bound electrons in a uniform electric field. Phys. Rev. B 1973, 8, 5579-5586. [CrossRef]

23. Holthaus, M.; Hone, D.W. Localization Effects in Ac-Driven Tight-Binding Lattices. Phil. Mag. B 1996, 74, 105-137. [CrossRef]

24. Girlando, A.; Painelli, A. Regular-dimerized stack and neutral-ionic interfaces in mixed-stack organic charge-transfer crystals. Phys. Rev. B 1986, 34, 2131-2139. [CrossRef] [PubMed]

25. Painelli, A.; Girlando, A. Zero-temperature phase diagram of mixed-stack charge-transfer crystals. Phys. Rev. $B$ 1988, 37, 5748-5760. [CrossRef] [PubMed]

26. Huai, P.; Zheng, H.; Nasu, K. Theory for Photoinduced Ionic-Neutral Structural Phase Transition in Quasi One-Dimensional Organic Molecular Crystal TTF-CA. J. Phys. Soc. Jpn. 2000, 69, 1788-1800. [CrossRef]

27. Soos, Z.G.; Painelli, A. Metastable domains and potential energy surfaces in organic charge-transfer salts with neutral-ionic phase transitions. Phys. Rev. B 2007, 75, 155119. [CrossRef]

28. Special Issue “The Neutral-Ionic Phase Transition". Crystals 2017. Edited by Painelli, A., Girlando, A. Available online: https://www.mdpi.com/journal/crystals/special_issues/The_neutral_ionic_phase_ transition (accessed on 10 May 2019).

29. Torrance, J.B.; Vazquez, J.E.; Mayerle, J.J.; Lee, V.Y. Discovery of a Neutral-to-Ionic Phase Transition in Organic Materials. Phys. Rev. Lett. 1981, 46, 253-257. [CrossRef]

30. Torrance, J.B.; Girlando, A.; Mayerle, J.J.; Crowley, J.I.; Lee, V.Y.; Batail, P.; LaPlaca, S.J. Anomalous Nature of Neutral-to-Ionic Phase Transition in Tetrathiafulvalene-Chloranil. Phys. Rev. Lett. 1981, 47, 1747-1750. [CrossRef]

31. Jacobsen, C.S.; Torrance, J.B. Behavior of charge-transfer absorption upon passing through the neutral-ionic phase transition. J. Chem. Phys. 1983, 79, 1075-1085. [CrossRef]

32. Le Cointe, M.; Lemée-Cailleau, M.H.; Cailleau, H.; Toudic, B.; Toupet, L.; Heger, G.; Moussa, F.; Schweiss, P.; Kraft, K.H.; Karl, N. Symmetry breaking and structural changes at the neutral-to-ionic transition tetrathiafulvalene-p-chloranil. Phys. Rev. B 1995, 51, 3374-3386. [CrossRef]

33. Lemée-Cailleau, M.H.; Le Cointe, M.; Cailleau, H.; Luty, T.; Moussa, F.; Roos, J.; Brinkmann, D.; Toudic, B.; Ayache, C.; Karl, N. Thermodynamics of the Neutral-to-Ionic Transition as Condensation and Crystallization of Charge-Transfer Excitations. Phys. Rev. Lett. 1997, 79, 1690-1693. [CrossRef]

34. Luty, T.; Cailleau, H.; Koshihara, S.; Collet, E.; Takesada, M.; Lemeé-Cailleau, M.H.; Cointe, M.B.L.; Nagaosa, N.; Tokura, Y.; Zienkiewicz, E.; Ouladdiaf, B. Static and Dynamic Order of Cooperative Multi-Electron Transfer. Europhys. Lett. 2002, 59, 619-625. [CrossRef]

35. Kobayashi, K.; Horiuchi, S.; Kumai, R.; Kagawa, F.; Murakami, Y.; Tokura, Y. Electronic Ferroelectricity in a Molecular Crystal with Large Polarization Directing Antiparallel to Ionic Displacement. Phys. Rev. Lett. 2012, 108, 237601. [CrossRef] [PubMed]

36. Katan, C.; Koenig, C.; Blöchl, P.E. Ab-Initio Calculations of One-Dimensional Band Structures of Mixed-Stack Molecular Crystals. Solid State Commun. 1997, 102, 589-594. [CrossRef]

37. Oison, V.; Katan, C.; Rabiller, P.; Souhassou, M.; Koenig, C. Neutral-ionic phase transition: A thorough ab-initio study of TTF-CA. Phys. Rev. B 2003, 67, 035120. [CrossRef]

38. Miyashita, S.; Tanaka, Y.; Iwai, S.; Yonemitsu, K. Charge, Lattice, and Spin Dynamics in Photoinduced Phase Transitions from Charge-Ordered Insulator to Metal in Quasi-Two-Dimensional Organic Conductors. J. Phys. Soc. Jpn. 2010, 79, 034708. [CrossRef] 
39. Gagliano, E.R.; Balseiro, C.A. Dynamical Properties of Quantum Many-Body Systems at Zero Temperature. Phys. Rev. Lett. 1987, 59, 2999-3002. [CrossRef]

40. Koshihara, S.; Tokura, Y.; Mitani, T.; Saito, G.; Koda, T. Photoinduced valence instability in the organic molecular compound tetrathiafulvalene-p-chloranil (TTF-CA). Phys. Rev. B 1990, 42, 6853-6856. [CrossRef]

41. Collet, E.; Lemée-Cailleau, M.H.; Cointe, M.B.L.; Cailleau, H.; Wulff, M.; Luty, T.; Koshihara, S.; Meyer, M.; Toupet, L.; Rabiller, P.; et al. Laser-Induced Ferroelectric Structural Order in an Organic Charge-Transfer Crystal. Science 2003, 300, 612-615. [CrossRef]

42. Guerin, L.; Collet, E.; Lemée-Cailleau, M.H.; Cointe, M.B.L.; Cailleau, H.; Plech, A.; Wulff, M.; Koshihara, S.Y.; Luty, T. Probing Photoinduced Phase Transition in a Charge-Transfer Molecular Crystal by 100 Picosecond X-Ray Diffraction. Chem. Phys. 2004, 299, 163-170. [CrossRef]

43. Katsura, H.; Sato, M.; Furuta, T.; Nagaosa, N. Theory of the Optical Conductivity of Spin Liquid States in One-Dimensional Mott Insulators. Phys. Rev. Lett. 2009, 103, 177402. [CrossRef] [PubMed]

44. Huang, Z.; Chen, L.; Zhao, Y. Transient Dynamics of a One-Dimensional Holstein Polaron under the Influence of an External Electric Field. Ann. Phys. (Berlin) 2017, 529, 1600367. [CrossRef]

(C) 2019 by the author. Licensee MDPI, Basel, Switzerland. This article is an open access article distributed under the terms and conditions of the Creative Commons Attribution (CC BY) license (http://creativecommons.org/licenses/by/4.0/). 